\title{
Psuedo-Reaction in Leprosy due to Gonorrhoea.
}

\author{
Donald P. Dow and John S. Narayan.
}

$\mathrm{A}$ MONG the diseases coincident with leprosy gonorrhoea is one of the most common and yet one of the most neglected. Being impressed with the importance of the disease as a complicating factor in the ordinary treatment of the inmates here, we were led to open a venereal clinic in the hospital. During the past year we have treated a great many cases and were interested to find that gonorrhoea either acute or chronic was ten times as common as syphilis. We are convinced that after treatment of the gonorrhoea many patients make rapid progress in their leprotic treatment, but in this paper we wish to draw attention, not to gonorrhoea or its treatment which are quite well known, but to a complication of the disease which is very liable to be overlooked, especially in leprosy, namely gonorrhoeal arthritis with its accompanying signs and symptoms. From our experience here we are led to believe that a proportion of so called leprareactions are in reality gonorrhoeal in nature.

Gonorrhoeal-arthritis is now generally regarded as due to a metastasis, the gonococcus being carried by the blood stream to the affected joint. The condition is more apt to occur in chronic gonorrhoea, or at any rate in the later stage of an acute attack, but it may occur, especially the polyarticular variety, as early as the third day.

Two varieties are met with (1) Acute (2) Chronic.

1. Acute. The acute type may manifest itself as:-

(a) An acute arthritis occurring in one joint only, though it may occasionally occur in two or three. The joint is swollen and tender, the skin surface red and shiny, and the joint affected acutely tender on movement and palpation.

(b) A poly-arthritis generally sub-acute, and affecting chiefly the smaller joints of the hand and foot. The joints are tender, but not so red and swollen as in the previous type.

(c) A diffuse arthralgia with no clinical signs in the joint itself but the affected joints are painful, and the pain is flitting.

2. Chronic. The chronic type may manifest itself as:- 


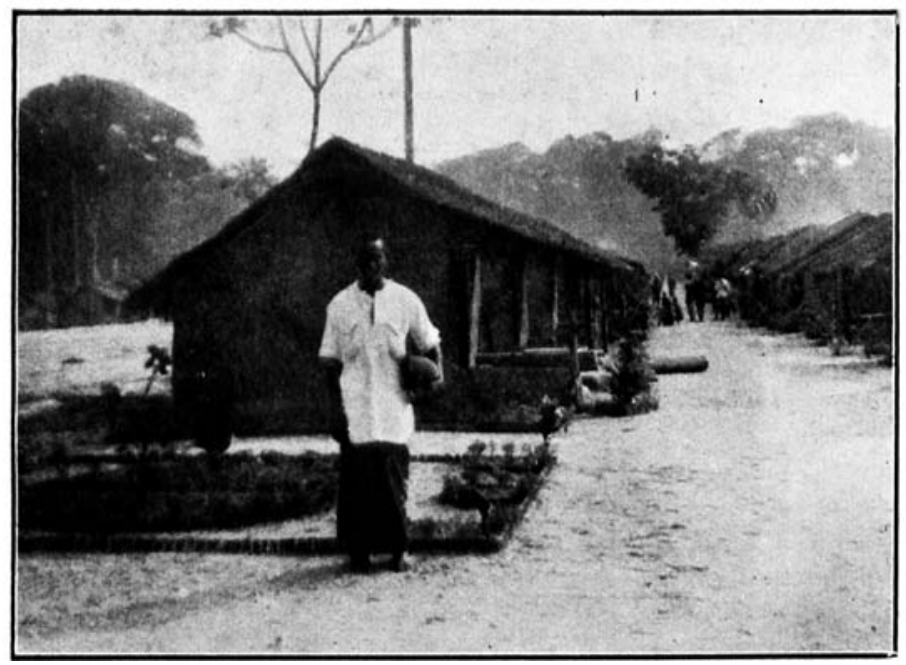

A Street in tile Men's Village, Itu leprosy Colony.

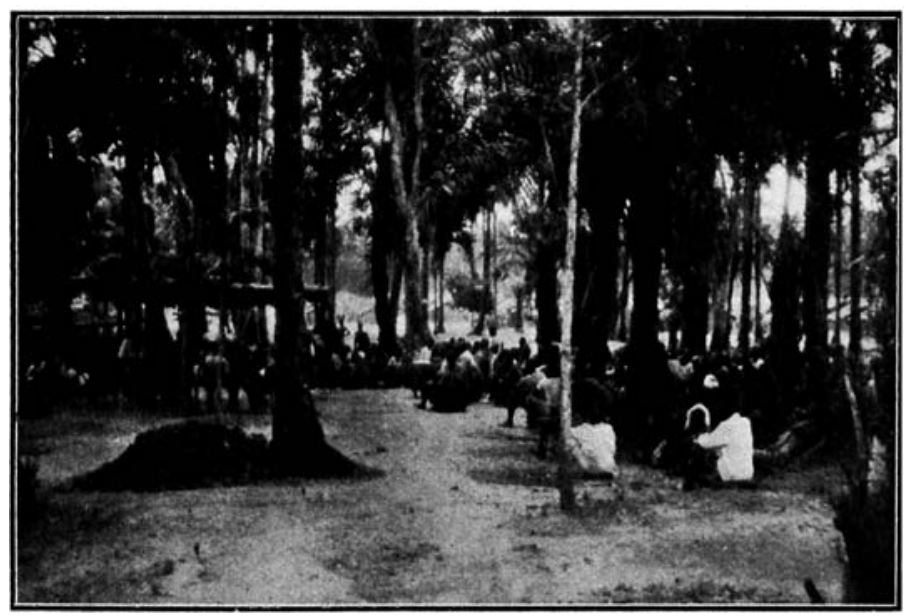

An Open-air Service at Itu Leprosy Colony.

(SEE DR. ARMSTRONG'S ARTICLE, PAGE 155) 


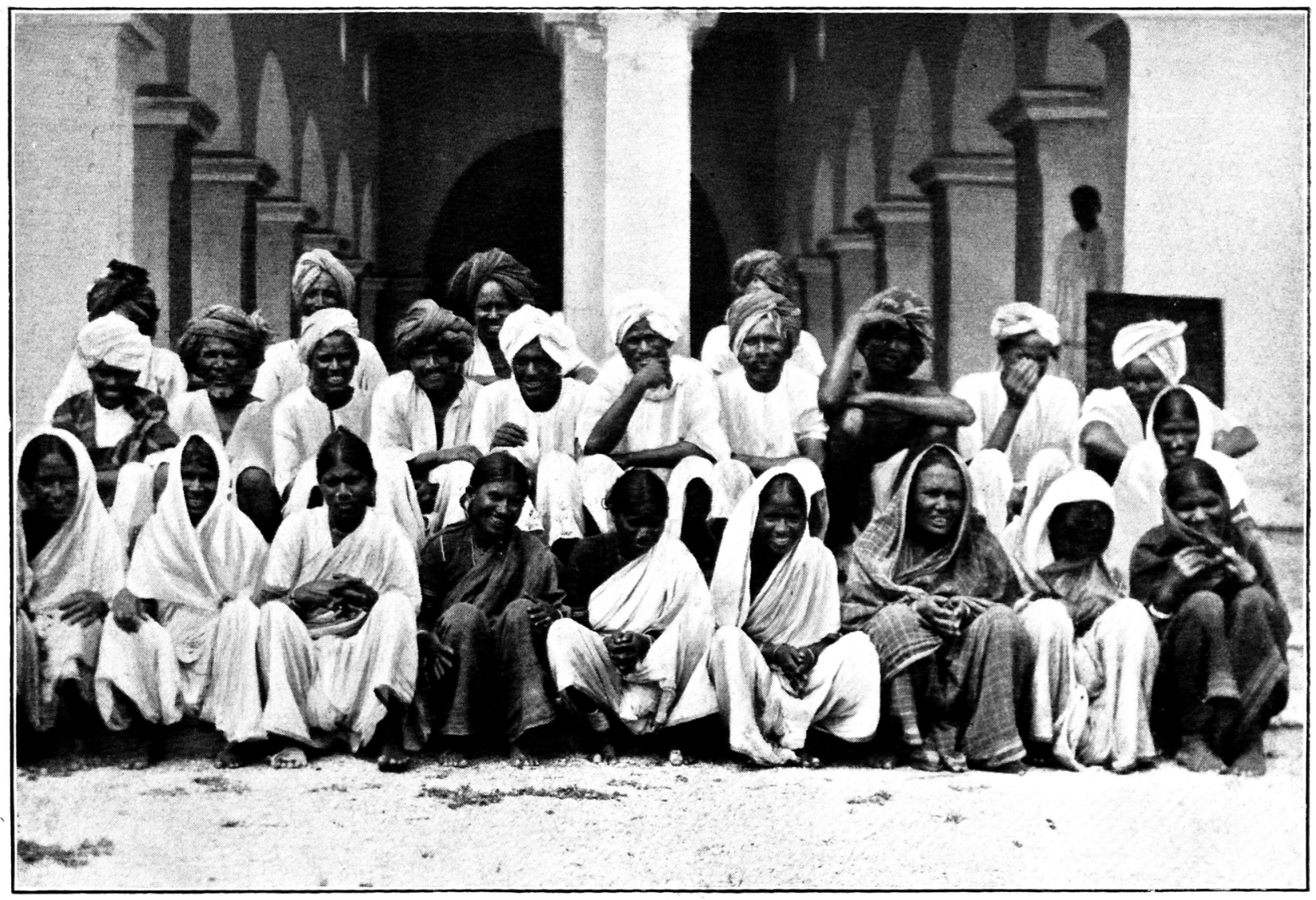

Group of "Symptom Free" Patients at Dichpali.

By permission of "The Kingdom Ozerseas." 
(a) A hydrops articuli usually affecting the knee joint. The affected joint is swollen and tense but not painful.

(b) An osteo-arthritis due to fibrinous exudate with subsequent adhesions.

The acute types are more common during the acute or subacute attacks of gonorrhoea; the chronic types follow a chronic infection of the posterior urethra or prostate.

With the chronic manifestations of gonorrhoeal infection of joints we are not at the moment concerned, but we wish to illustrate by the following series of cases the similarity between acute and sub-acute gonorrhoeal arthritis and lepra reaction.

Case A.E., Male, age 35 years, C.1., was admitted to the ward with left ulnar nerve reaction and slight pain in the wrist joints on 15-11-33, and was treated with ephedrine, salicytes, and gelsemium; the nerve pain was relieved, but this was followed by tenderness and pain in all the joints. The above drug treatment did not give relief and the patient was in excruciating agony. No local application brought any relief. He denied gonorrhoeal infection; but he was examined, and a smear taken was found to be positive for gonococci. Since the case was now regarded as one of gonorrhoeal arthritis, two injections of atophonyle 10 c.c. each were given intravenously bi-weekly, and an alkaline mixture orally, and after one week all the pains in the joints disappeared. The swelling of the right knee did not however subside; so we later drew off 8 c.c. of yellowish fluid. Two weekly injections of hexamine, grains 5 , dissolved in 5 c.c. distilled water were given intravenously. The patient was relieved of his pains and was discharged from the ward on 1-1-34. He has been given urethral irrigations and prostatic massage because of urethral discharge. The patient later admitted that he had been infected 8 years before by direct contact and a year later his leprosy developed.

Case C.M., Male, age 40, C.2., frequently suffered from skin reaction accompanied by pains in the joints of the hands and feet. He volunteered a history of gonococcal infection 6 years previously, and a year subsequently developed leprosy. There was an abundant discharge of pus per urethram. The fore-skin was enlarged, swollen and oedematous. There was burning sensation and difficulty of micturition. This man developed a skin reaction and joint nains. Routine treatment of lepra reaction was without effect on the joint pains. 
On being treated for gonococcal arthritis the joint pains subsided and he was discharged well. The anti-gonococcal treatment was continued.

Case C.V., male, age 26, N.1., was admitted to the hospital on 8-11-33 with no history of venereal disease, but kahn 4 plus, therefore he was put on anti-syphilitic treatment under which he did well. This patient was admitted to the wards on 4-12-33 complaining of joint pains generally. He denied the possibility of gonorrhoeal infection, but in view of the number of cases of arthritis simulating lepra reaction we insisted on an examination when a copious discharge of pus per urethram was found, and the prepuce was swollen and tender; a smear was highly positive for gonorrhoea. Anti-gonococcal treatment cleared up the condition.

Case B, male, age 28, C.1., shortly after admission complained of pain in both knee joints which were regarded as leprotic in nature, but treatment along ordinary lines was of no avail; therefore he was examined for gonorrhoea and a urethral discharge of pus was found. He admitted gonococcal infection 6 years before, and a year later developed leprosy. The treatment of the gonorrhoea cleared off the joint pains.

Case O.K., male, age 40, C.2., was admitted on 30-7-33. $\mathrm{He}$ was getting the usual leprotic treatment, but after 3 months he complained of knee joint pains which he attributed to the anti-leprotic injections. Treatment of this affection on anti-leprotic lines was without any benefit. He gave no history of gonorrhoea, but during the examination of a batch of patients for gonorrhoea he was discovered to have a copious urethral discharge. Therefore anti-gonococcal treatment was given with very heneficial results. Later he admitted gonorrhoeal infection 5 years previously, followed by leprosy $1 \frac{1}{2}$ years later.

All of the foregoing cases were complaining of symptoms referable to leprosy in a reacting stage and there are a great many such cases which we are treating here. The first three cases all had signs of an acute lepra reaction (fever, malaise, joint pains, body pains with rise in sedimentation index) while the last two were of a more chronic nature but they also had some signs and symptoms referable to the disease for which they were admitted here.

\section{Conclusions.}

From our observations we are led to believe :-

1. The gonorrhoeal arthritis with its accompaniments is far more 


\section{LeProsy ReVieW}

common than is generally supposed and may very easily be mistalicn for lepra reaction.

2. Untreated gonorrhoca seems to persist for long periods in many people and while we have cloubts that many of the patients here have been reinfected we belicve that the disease persists in a-sub-acute form for a surprisingly long period.

3. Almost every patient whom we have treated gave the history of leprosy having manifested itself within two years of the gonorrhneal infection and we are led to wonder if the debility which frequently follows such an infection has not sufficiently lowered the resistance of the patient to allow the leprosy to become clinically manifest, for in none of our cases were we successful in eliciting a history of any ther debilitating disease.

4. In some cases here of true lepra-reaction we find that the patients make no progress whatever until the coinciclent gonorrhoen is treated. But immediately that is done the repeated reactions to which such patients are subject subside, and progress commonly takes place. 